\title{
Comparison of different laboratory tests in the evaluation of hemorrhagic risk of patients using rivaroxaban in the critical care setting: diagnostic accuracy study
}

Marjorie Paris Colombini ${ }^{* *}$, Priscilla Bento Matos Cruz Derogis ${ }^{1}$, Valdir Fernandes de Aranda ${ }^{1}$, João Carlos de Campos Guerra', Nelson Hamerschlak ${ }^{2}$ and Cristóvão Luis Pitangueiras Mangueira ${ }^{1}$

\begin{abstract}
Background: Rivaroxaban is a direct oral anticoagulant designed to dispense with the necessity of laboratory monitoring. However, monitoring rivaroxaban levels is necessary in certain clinical conditions, especially in the critical care setting.

Methods: This is a diagnostic accuracy study evaluating sensitivity and specificity of prothrombin time (PT), activated partial thromboplastin time (aPTT), and Dilute Russell viper venom time (dRVT), to evaluate the hemorrhagic risk in patients taking rivaroxaban. The study used a convenience sample of 40 clinically stable patients using rivaroxaban to treat deep vein thrombosis or atrial fibrillation admitted in a private hospital in Brazil, compared to a group of 60 healthy controls. The samples from patients were collected two hours after the use of the medication (peak) and two hours before the next dose (trough).

Results: The correlation with the plasmatic concentration measured by anti-FXa assay was higher for PT and dRVTS. The PT and aPTT tests presented higher specificity, while dRVT was 100\% sensible.

Conclusions: There was a strong correlation between the tests and the plasma concentration of the drug. Additionally, our results demonstrated the potential use of dRWT as a screening test in the emergency room and the need of a second test to improve specificity.
\end{abstract}

Keywords: Anticoagulants, Direct oral anticoagulants, Rivaroxaban, Clinical laboratory techniques, Prothrombin time, Russell's viper venom time

\section{Background}

The traditional anticoagulant drugs that exist for the prevention and treatment of venous thromboembolism (VTE), prevention of cerebral vascular accident in patients with atrial fibrillation (AF), and secondary prevention in patients with acute coronary syndrome (ACS) require constant laboratory monitoring, which is sometimes burdensome and inconvenient for the patient.

\footnotetext{
* Correspondence: marjorie@einstein.br

${ }^{1}$ Department of Diagnostic and Preventive Medicine and Clinical Laboratory, Hospital Israelita Albert Einstein, São Paulo, Brazil

Full list of author information is available at the end of the article
}

Rivaroxaban is a direct oral anticoagulant (DOAC) that dispenses with this type of control. It is an antithrombotic drug, that acts directly inhibiting activated factor $\mathrm{X}$, impeding the generation of thrombin, and consequently preventing the formation of clots. It also has the advantage that it can be administered in a single daily dose [1].

Rivaroxaban has high bioavailability after oral administration, with a maximum peak of action at around 1.5 to $2 \mathrm{~h}$ after use (peak action), a mean half-life of between 5 and $9 \mathrm{~h}$ in young patients and 12 to $13 \mathrm{~h}$ in those aged over 75 years. It is eliminated in two ways: two thirds are metabolized by the liver (via CYP3A4 and CYP2J2) 
without any circulating active metabolite identified, and one third is excreted unaltered in the urine [2, 3].

Although direct oral anticoagulants have been designed to dispense the necessity of laboratory monitoring, the literature has demonstrated that this monitoring is potentially useful in certain conditions $[2,4,5]$.

So far, it is known that rivaroxaban can prolong the times in the conventional clotting tests used to evaluate the risk of hemorrhage, such as prothrombin time (PT), activated partial thromboplastin time (aPTT), and thrombin time (TT), but it has not yet been possible to establish a therapeutic window of clinical interest that can be linearly correlated with the plasma concentration of the drug for these tests. The only two specific tests available to date, and that present correlation with the plasma concentration of the drug, are anti-FXa assay (indirect method) [1, 4], and liquid chromatography/mass spectrometry (direct method).

More recently, some authors have considered PT $[6,7]$ and dilute Russell viper venom time (dRVVT) [8-11] as the most promising tests for this type of monitoring. dRVVT evaluates only the common laboratory coagulation pathway test (factors $\mathrm{X}, \mathrm{V}, \mathrm{II}$ and I) after activation of factor $\mathrm{X}$ by Russell viper venom, minimizing the possibility of interference of dysfunction of the other clotting factors. There are also two different types of reagents in terms of the concentration of phospholipid used: the screen test (lowest concentration of phospholipid, dRVVTS) and the confirm test (highest concentration, dRVVTC) to evidence the presence of lupus anticoagulant. Exner et al., suggested that dRVVT could possibly be used to detect and maybe determine the plasmatic concentration of many anticoagulants, including rivaroxaban [11]. Douxfils et al. state also that the "russell viper venom time test" allows a rapid estimation of the intensity of anticoagulation mediated by rivaroxaban, although the authors did not differentiate the drugs type [12].

Altman and Gonzalez published a study in which they proposed that Russell's viper venom is the most sensitive for identifying patients at risk of hemorrhage or exhibiting low anticoagulant effect, but they emphasize the need for other studies, to establish the sensitivity of other methods and identify cut-off values [8].

\section{Objective}

The primary objective of our work was to compare the sensitivity, specificity, positive predictive value (PPV) and negative predictive value (NPV) of PT, aPTT and dRVVT, in order to exclude qualitatively the plasma concentrations that are relevant in critical care. The secondary objective was to correlate PT, aPTT, dRVVT (screen and confirm) assays with plasmatic concentration of the drug measured by the anti-Xa methodology in the same patients.

\section{Methods \\ Study design, setting and ethics}

This is a diagnostic observational study, conducted at a private hospital in São Paulo, Brazil, with a convenience sample of stable patients using the drug rivaroxaban.

The Institutional Review Board approved the protocol in advance under CAAE number: 34661614.1.0000.0071. All the subjects signed an informed consent form to perform blood tests and to participate in the study.

\section{Subjects}

The subjects of this study are all patients consecutively admitted in the period of September 22, 2014 to December 21,2015 , in daily use of rivaroxaban at daily doses of 10 , 15 or $20 \mathrm{mg}$ daily (single dose), due to diagnosed thrombosis of the lower limbs, or those at risk of embolism due to atrial fibrillation. Patients with creatinine clearance lower than $15 \mathrm{~mL} / \mathrm{min}$ or using rivaroxaban twice-daily regiment were excluded.

Plasma of 60 healthy controls without any known defects in the blood coagulation was used to estimate the normal range for the PT, aPTT and dRVVT screen and confirm assays.

\section{Laboratorial analytical methods}

The patients' blood samples were collected on two separate occasions: two hours after the use of the medication ("peak" moment) and two hours before the next dose ("trough" moment). The samples were collected according to the norms recognized by the Clinical Laboratory of the hospital, in test tubes containing 3.2\% sodium citrate anticoagulant (Sarstedt, Newton, NC). Conventional PT tests were performed with the reagent STA Neoplastine CI Plus 10 and aPTT with STA Cephascreen 4 and processed on a Stago STA-R Evolution coagulation analyzer (Stago, Asnières-sur-Seine, France).

The Russell viper venom test was processed with the reagent STA Staclot DRVV (Screen and Confirm; Stago, Asnières-sur-Seine, France), at different phospholipid concentrations; one low, designated dRVVTS, and the other with a high concentration, designated dRVVTC.

The evaluation of anti-FXa assay was performed with the reagent STA-Liquid Anti-FXa with specific calibrator for rivaroxaban (Stago, Asnières-sur-Seine, France).

\section{Definitions}

In this study, the following definitions were used:

- $\leq 30 \mathrm{ng} / \mathrm{mL}$ as the plasma concentration cut-off defined as safe for invasive procedures [13, 14]; 
- $\leq 50 \mathrm{ng} / \mathrm{mL}$ as the plasma concentration indicating moderate risk cut-off defined by Lim et al. [15] and Levy et al. [16];

- $\leq 100 \mathrm{ng} / \mathrm{mL}$ as the concentration that may lead to thrombolysis in ischemic stroke [14, 17];

- sensitivity as the proportion of individuals with a higher plasma concentration than the threshold (30 $\mathrm{ng} / \mathrm{mL}, 50 \mathrm{ng} / \mathrm{mL}$ or $100 \mathrm{ng} / \mathrm{mL}$ ) and a test result above normality;

- specificity as the proportion of individuals with a plasma concentration lower than the threshold (30 ng/mL, $50 \mathrm{ng} / \mathrm{mL}$ or $100 \mathrm{ng} / \mathrm{mL}$ ) and a test result within normality range;

- positive predictive value (PPV) as the proportion of individuals with a positive test result who actually present plasma concentrations higher than the threshold (30 ng/mL, $50 \mathrm{ng} / \mathrm{mL}$ or $100 \mathrm{ng} / \mathrm{mL}$ );

- negative predictive value (NPV) as the proportion of individuals with a negative test result who do really present plasma concentrations lower than the threshold (30 ng/mL, $50 \mathrm{ng} / \mathrm{mL}$ or $100 \mathrm{ng} / \mathrm{mL}$ );

- correlation grades: coefficients $<0.2$ meaning very weak correlation; 0.2 to 0.39 as weak correlations; 0.40 to 0.59 as moderate; 0.60 to 0.79 strong correlation; > 0.80 very strong [18].

\section{Statistical analysis}

The plasmatic concentrations were described as means, standard deviation and 95\% confidence intervals (CI). Spearman's correlations were calculated between the anti-FXa assay values (above the lower limit of quantitation, LOQ, $25 \mathrm{ng} / \mathrm{mL}$ ) and the laboratory tests of interest. The results were illustrated using scatter plots. The analyses were performed with the software GraphPad Prism 5 (GraphPad Software, San Diego, CA), considering a level of significance of 0.05. The results from healthy subjects were analyzed using the EP Evaluator 11.1.0.26 (Data Innnovations, LLC) software program. EP Evaluator follows the recommendations of the CLSI C28-A (Clinical and Laboratory Standards Institute guideline) [19]. The normal ranges were expressed in the $90 \% \mathrm{CI}$. The diagnostic test evaluation was calculated using the free online MEDCALC easy-to-use statistical software. CI for sensitivity and specificity are "exact" Clopper-Pearson CI; and, for the predictive values, the standard logit CI given by Mercaldo et al. [20] were used.

\section{Results}

\section{Study group}

In the study period, 40 patients required the use of anticoagulation and were included and evaluated (Table 1).
Table 1 Patients' baseline data

\begin{tabular}{ll}
\hline Gender & $\mathrm{n}(\%)$ \\
Female & $20(50)$ \\
Male & $20(50)$ \\
Rivaroxaban dosage & $\mathrm{n}(\%)$ \\
$10 \mathrm{mg} /$ day & $12(30.0)$ \\
$15 \mathrm{mg} /$ day & $19(47.5)$ \\
$20 \mathrm{mg} /$ day & $9(22.5)$ \\
Age & \\
Average (standard deviation) & $69(21)$ \\
Minimum and maximum age & $27-95$ \\
\hline
\end{tabular}

\section{Plasmatic concentration}

The plasmatic concentration calculated by anti-FXa assay were as expected in trough, mainly less than the LOQ $(25 \mathrm{ng} / \mathrm{mL})$, for all dosages. In peak time, the results were: $63.4 \pm 61.0 \mathrm{ng} / \mathrm{mL}$ (mean \pm standard deviation, SD, with 95\% CI: 24.7-102.1) for $10 \mathrm{mg}$; $142.5 \pm 93.2 \mathrm{ng} / \mathrm{mL}$ (CI: 97.6-187.4) for $15 \mathrm{mg}$, and $203.4 \pm 85.6 \mathrm{ng} / \mathrm{mL}$ (CI: 137.6-269.2) for $20 \mathrm{mg}$.

\section{Correlation between different methods with plasmatic concentration}

All results of PT, dRVVT screen and confirm (Figs. 1, 2 and 3) presented a strong positive correlation with antiFXa assay $(r \geq 0.60)$. The correlation of aPTT (Fig. 4) was only moderate $(r=0.53)$.

\section{Normal range}

Table 2 shows the normal range calculated for TP, aPTT, dRVVs and dRVVc.

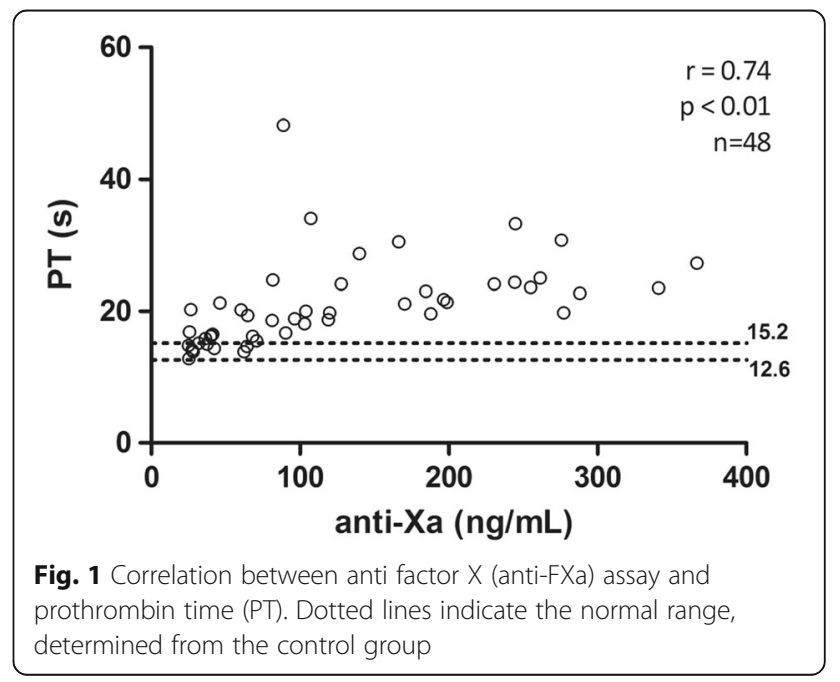




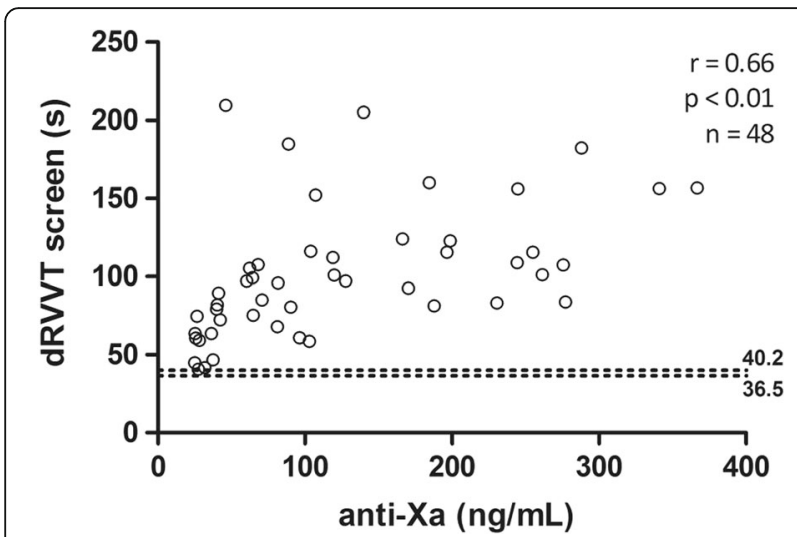

Fig. 2 Correlation between anti factor $X$ (anti-FXa) and dilute Russell viper venom time (dRWT) screen assays. Dotted lines indicate the normal range, determined from the control group

\section{Sensitivity, specificity, PPV and NPV}

Rivaroxaban concentration $<30 \mathrm{ng} / \mathrm{mL}$ was observed in $38 / 80$ samples; < $50 \mathrm{ng} / \mathrm{mL}$, in 46/80; and <100 ng/mL, in 57/80. Figs. 5, 6, 7, 8 and Tables 3, 4, 5 show the results dispersion and the performance of different tests according to different thresholds. It is possible to observe that, as the threshold increases, the TP and aPTT sensitivity also increases and specificity is reduced. dRVVs and dRVVc had the highest sensitivity regardless of the threshold adopted.

\section{Discussion}

Among the direct oral anticoagulants (DOACs), rivaroxaban has been widely used in medical practice, particularly because it dispenses with the necessity of laboratory control in the majority of patients $[4,5,8]$. However, in conditions where a laboratory evaluation is desirable, it has been demonstrated by many authors [1] that the test of choice, due to its high specificity, is the plasmatic concentration obtained by the chromogenic anti-FXa assay with specific calibrator for rivaroxaban.

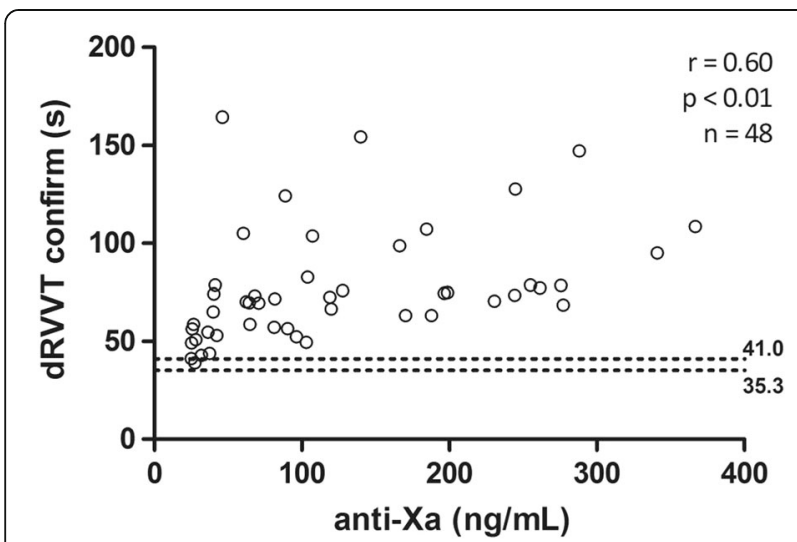

Fig. 3 Relationship between anti factor X (anti-FXa) and dilute Russell viper venom time (dRWT) confirm assays. Dotted lines indicate the normal range, determined from the control group

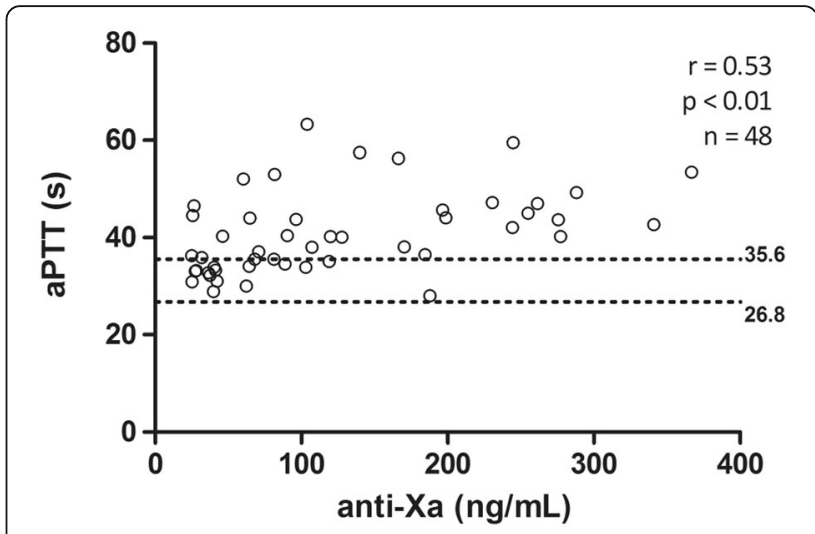

Fig. 4 Correlation between anti factor $X$ (anti-FXa) assay and activated partial thromboplastin time (aPTT). Dotted lines indicate the normal range, determined from the control group

According to Baglin et al. [21], three methodologies can be additionally used for rivaroxaban monitoring: activated partial thromboplastin time (aPTT), prothrombin time (PT) and plasmatic concentration determination. Recent data pointed dRVVT as an additional tool $[8,9]$.

Levy et al. [10] cited that is very important to know when the last dose of the direct oral anticoagulant was taken by the patient in order to determine whether the levels are likely to increase or fall over time. There are some situations, however, in that obtaining this information is impossible, and this happens frequently in the emergency setting [14]. Besides, some patients might metabolize anticoagulant drugs differently. Therefore, it is important to know the performance of the evaluation techniques that are available in the laboratory, so that a correct approach can be used in the emergency room. Because of that, this study sought to evaluate the correlation and performance of different tests regarding the plasmatic concentration of rivaroxaban.

The plasmatic concentrations measured by anti-FXa assay were comparable to Mueck et al. [22]. Our results also show that PT was most closely correlated to plasmatic concentration measured by anti-FXa assay, followed by dRVVTs, dRVVTc and aPTT. The PT and aPTT correlation to plasmatic concentration was strongly discussed by Francart et al. [23], depending on reagent type. Douxfils et al. studied the response of two PT reagents and correlated PT data to the plasma concentration measured by

Table 2 Normal range calculated using samples from 60 health volunteers

\begin{tabular}{lll}
\hline Tests & $\begin{array}{l}\text { Lower limit }(90 \% \\
\text { confidence interval) }\end{array}$ & $\begin{array}{l}\text { Upper limit }(90 \% \\
\text { confidence interval) }\end{array}$ \\
\hline PT (seconds) & $12.6(12.37-12.85)$ & $15.2(14.96-15.44)$ \\
aPTT (seconds) & $26.8(26.1-27.5)$ & $35.6(34.7-36.6)$ \\
dRWT screen (seconds) & $36.5(36.2-36.9)$ & $40.2(39.9-40.6)$ \\
dRWT confirm (seconds) & $35.3(34.8-35.8)$ & $41.0(40.4-41.5)$ \\
\hline
\end{tabular}




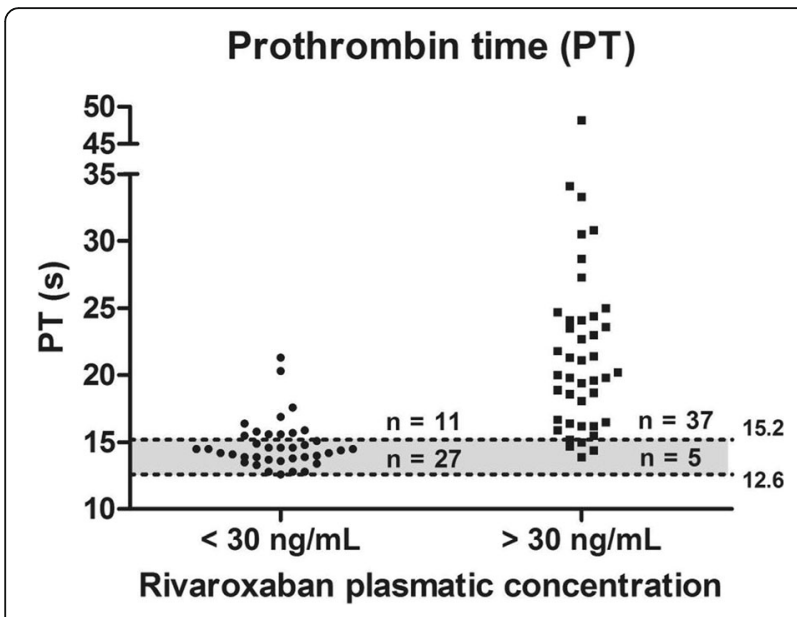

Fig. 5 Prothrombin time (PT; seconds) distribution according to rivaroxaban plasmatic concentration. Normal range is represented by the gray band with a lower limit of $12.6 \mathrm{~s}$ (90\% confidence interval, $\mathrm{Cl}$ : $12.37-$ 12.85) and upper limit of 15.2 s (90\% Cl: 14.96-15.44)

HPLC-MS/MS. Correlation was found to be very strong (0.86) [9]. The differences between theirs and our results can be attributed to the characteristics of the patients evaluated, the reagents used and the methodology used in the plasmatic concentration measurement.

Gosselin et al. [10] correlated dRVVT to plasmatic concentration obtained by the direct method of liquid chromatography/mass spectrometry. They used the Siemens LA2 and Precision Biologics DRVVT reagents and presented a higher correlation coefficient (0.85 and 0.88 , depending on the reagent). As for the PT and aPTT assays, the differences found can be attributed to the reagents, methods and even patients evaluated. In our study, if we isolate the results obtained with patients using $20 \mathrm{mg} /$ day

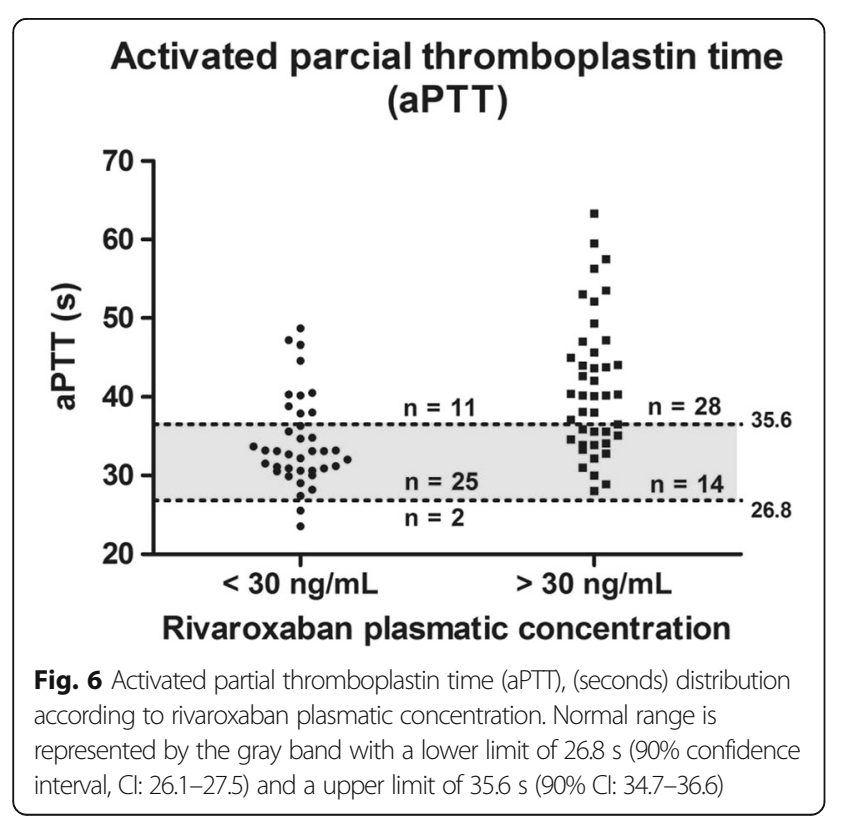

\section{Dilute Russell viper venom time screen (dRVVTS)}

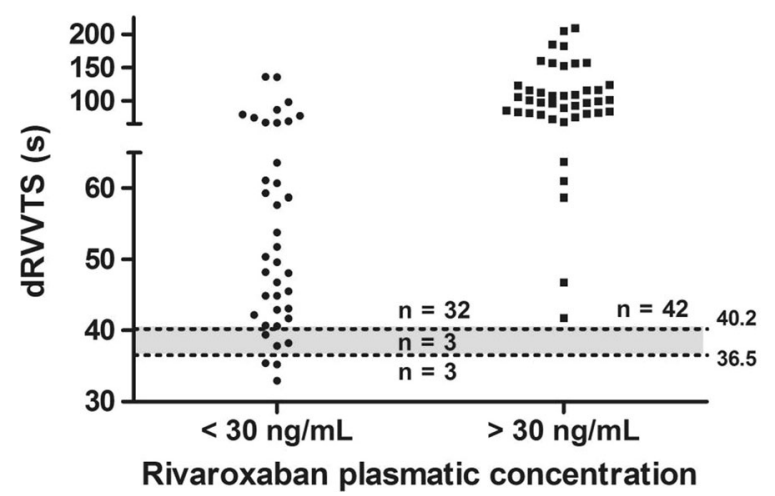

Fig. 7 Dilute Russell viper venom time screen (dRWTS) (seconds) distribution according to rivaroxaban plasmatic concentration. Normal range is represented by the gray band with a lower limit of 36.5 s (90\% confidence interval, Cl: $36.2-36.9)$ and upper limit of 40.2 s (90\% Cl: 39.9-40.6)

$(n=18)$, the correlation coefficient with anti-FXa assay would be 0.82 .

Ebner et al. [14] recently described two thresholds of plasma concentration that are relevant in situations of emergency (30 and $100 \mathrm{ng} / \mathrm{mL}$ ). Levy et al. [16] added the threshold of $50 \mathrm{ng} / \mathrm{mL}$ in cases of patients with severe bleeding. Lim et al., in 2016, described the same cut-off of $50 \mathrm{ng} / \mathrm{mL}$ for rivaroxaban, apixaban and dabigatran [15]. For this reason, the diagnostic test evaluation in our study was conducted using the three thresholds ( $<30 \mathrm{ng} / \mathrm{mL},<50 \mathrm{ng} / \mathrm{mL}$ and $<100 \mathrm{ng} / \mathrm{mL}$ ).

Sensitivity and specificity of PT and aPTT were threshold-dependent. PT is indeed frequently described as

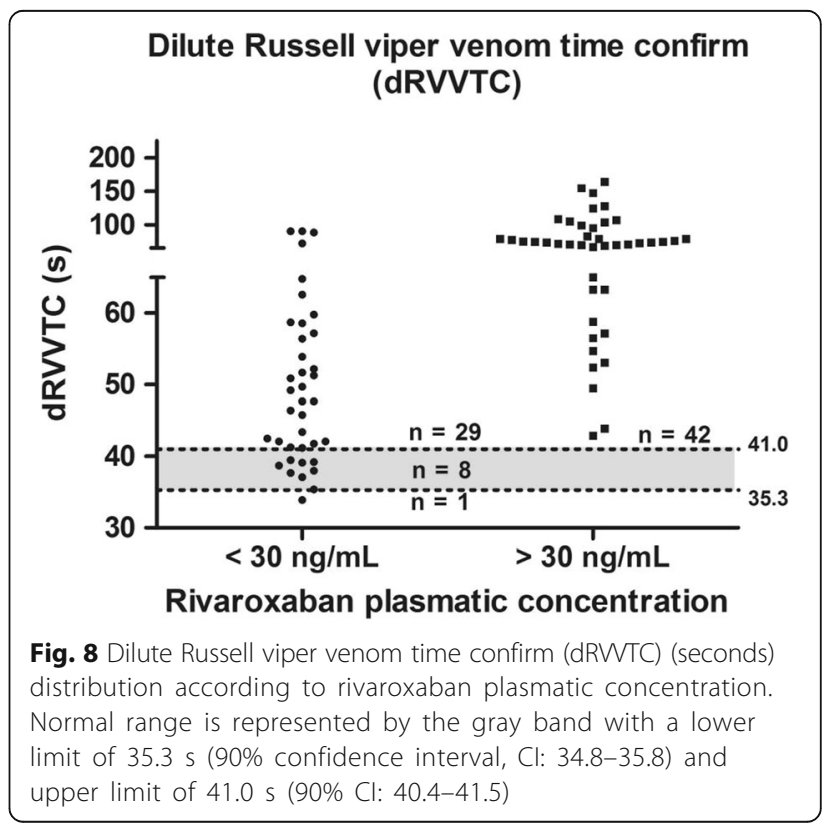


Table 3 Performance of four different tests for rivaroxaban monitoring (threshold: $30 \mathrm{ng} / \mathrm{mL}$ )

\begin{tabular}{lllll}
\hline Test & $\begin{array}{l}\text { Sensitivity } \\
(\%, 95 \% \mathrm{Cl})\end{array}$ & $\begin{array}{l}\text { Specificity } \\
(\%, 95 \% \mathrm{Cl})\end{array}$ & $\begin{array}{l}\text { PPV } \\
(\%, 95 \% \mathrm{Cl})\end{array}$ & $\begin{array}{l}\text { NPV } \\
(\%, 95 \% \mathrm{Cl})\end{array}$ \\
\hline PT & $88(74-96)$ & $71(54-85)$ & $77(67-85)$ & $84(70-93)$ \\
aPTT & $67(50-80)$ & $71(54-85)$ & $72(60-81)$ & $66(55-76)$ \\
dRWs & $100(92-100)$ & $16(6-31)$ & $57(53-60)$ & 100 \\
dRWc & $100(92-100)$ & $24(11-40)$ & $59(41-64)$ & 100 \\
\hline
\end{tabular}

$P P V$ positive predictive value, $N P V$ negative predictive value, $\mathrm{Cl}$ confidence interval

an adequate test for rivaroxaban monitoring $[4,5,24,25]$. Lim et al., in 2016, using the same reagent for PT assay, demonstrated a sensitivity and NPV above $90 \%$ for the cut-off of $50 \mathrm{ng} / \mathrm{mL}$ [15]. These results corroborate our findings. However, the observed differences in specificity and PPV can be attributed to the group studied. In addition to this influence, we believe that the differences in diagnostic accuracy related to aPTT may depend on the reagent used.

dRVVT screen and confirm tests presented maximum sensitivity and NPV, i.e., 100\%, regardless of the threshold used. This would undoubtedly the safest test for exclusion of plasmatic concentration associated to hemorrhagic risk when the results are normal. The number of false positive results in relation to the total of exams for dRVVT screen and confirm varied from $32 / 80$ to $51 / 80$. These results are similar to the literature $[7,26]$.

Gosselin et al. [27], Exner et al. [11] described dRVVT as highly sensible for the presence of rivaroxaban. In fact, the authors described the application of dRVVT for many DOACs. Douxfils et al. [12] discussed also that dRVVT could be useful to assess pharmacodynamics of DOACs, with the advantage that it is a single test applied to different DOACs.

Gosselin et al. [27] describe that anticoagulants cause false results not only in coagulometric tests, as in the investigation of lupus anticoagulant, for example, but also in chromogenic tests. The interference of rivaroxaban on dRVVT was also described by other authors [26, 28, 29]. It is important to highlight that the sensitivity and specificity of a quantitative test are dependent on the cut-off value above or below which the test is positive [30].

Table 4 Performance of four different tests for rivaroxaban monitoring (threshold: $50 \mathrm{ng} / \mathrm{mL}$ )

\begin{tabular}{lllll}
\hline Test & $\begin{array}{l}\text { Sensitivity } \\
(\%, 95 \% \mathrm{Cl})\end{array}$ & $\begin{array}{l}\text { Specificity } \\
(\%, 95 \% \mathrm{Cl})\end{array}$ & $\begin{array}{l}\text { PPV } \\
(\%, 95 \% \mathrm{Cl})\end{array}$ & $\begin{array}{l}\text { NPV } \\
(\%, 95 \% \mathrm{Cl})\end{array}$ \\
\hline PT & $94(80-99)$ & $65(50-79)$ & $67(57-75)$ & $94(79-98)$ \\
aPTT & $76(59-89)$ & $72(57-84)$ & $67(55-77)$ & $80(69-89)$ \\
dRWs & $100(90-100)$ & $13(5-26)$ & $46(43-49)$ & 100 \\
dRWc & $100(90-100)$ & $20(9-34)$ & $48(44-51)$ & 100 \\
\hline
\end{tabular}

$P P V$ positive predictive value, $N P V$ negative predictive value, $\mathrm{Cl}$ confidence interval
Table 5 Performance of four different tests for rivaroxaban monitoring (threshold: $100 \mathrm{ng} / \mathrm{mL}$ )

\begin{tabular}{lllll}
\hline Test & $\begin{array}{l}\text { Sensitivity } \\
(\%, 95 \% \mathrm{Cl})\end{array}$ & $\begin{array}{l}\text { Specificity } \\
(\%, 95 \% \mathrm{Cl})\end{array}$ & $\begin{array}{l}\text { PPV } \\
(\%, 95 \% \mathrm{Cl})\end{array}$ & $\begin{array}{l}\text { NPV } \\
(\%, 95 \% \mathrm{Cl})\end{array}$ \\
\hline PT & $100(85-100)$ & $56(42-69)$ & $48(41-55)$ & 100 \\
aPTT & $100(85-100)$ & $67(53-79)$ & $55(46-64)$ & 100 \\
dRWs & $100(85-100)$ & $11(4-22)$ & $31(29-33)$ & 100 \\
dRWc & $100(85-100)$ & $16(8-29)$ & $32(30-35)$ & 100 \\
\hline
\end{tabular}

$P P V$ positive predictive value, $N P V$ negative predictive value, $\mathrm{Cl}$ confidence interval

Based on our results, we suggest that the patients with hemorrhagic risk (those with dRVVT screen and confirm tests above normality, that have sensitivity and low specificity) be submitted to a second test with higher diagnostic specificity (anti-Xa test calibrated with rivaroxaban or, in the absence of this, PT). This would allow the reduction of false positives and inadequate therapeutic interventions.

The potential limitations of this study must be considered, specially the reduced and highly homogeneous and clinically stable sample of patients, which might not be the case of other emergency settings. This must be considered in light of the fact that PPV and NPV are highly dependent on the disease prevalence.

\section{Conclusions}

In conclusion, our findings showed that there is a correlation between the tests studied and the plasma concentration of rivaroxaban, confirming the existing description in the literature. In addition, as shown by the sensitivity and specificity results, our study suggests the applicability of the tests evaluated in the screening of plasmatic concentration associated to hemorrhagic condition in patients using rivaroxaban. However, it is crucial that the laboratory informs the attending physician about the diagnostic limitations of this group of tests in the evaluation of patients using anticoagulants, using an appropriate and clear note in the lab results report. Additionally, our results show a cut-off dependent behavior of the tests that must be better investigated in future studies.

\section{Abbreviations}

ACS: Acute coronary syndrome; AF: Atrial fibrillation; anti-FXa: Anti factor X; aPTT: Activated partial thromboplastin time; C: Confirmatory; dRWT: Dilute Russell viper venom time; PT: Prothrombin time; S: Screen; TT: Thrombin time; VTE: Venous thromboembolism

\section{Acknowledgements}

The authors thank Patricia Logullo for editorial assistance during manuscript preparation.

\section{Funding}

This study received no funding.

Availability of data and materials

Please contact author for data requests. 


\section{Authors' contributions}

MPC, VFA, JCCG, NH and CLPM designed and coordinated the study. MPC, PBMCD analyzed and interpreted data and drafted the manuscript. MPC, VFA and JCCG carried out the assays, interpreted data and drafted the manuscript. PBMCD performed the statistical analysis. All authors read and approved the final manuscript.

\section{Ethics approval and consent to participate}

The Institutional Review Board approved the study protocol (number: $34,661,614.1 .0000 .0071$ ). All the subjects signed informed consent forms to undergo blood tests and to participate in the study.

\section{Consent for publication}

Not applicable (the study will not be published with individual data).

\section{Competing interests}

The authors declare that they have no competing interests.

\section{Publisher's Note}

Springer Nature remains neutral with regard to jurisdictional claims in published maps and institutional affiliations.

\section{Author details}

'Department of Diagnostic and Preventive Medicine and Clinical Laboratory, Hospital Israelita Albert Einstein, São Paulo, Brazil. ${ }^{2}$ Department of Hematology, Hospital Israelita Albert Einstein, São Paulo, Brazil.

Received: 18 January 2017 Accepted: 26 June 2017

Published online: 15 August 2017

\section{References}

1. Tripodi A. The laboratory and the direct oral anticoagulants. Blood. 2013; 121(2):4032-5.

2. Leung LLK. Direct oral anticoagulants: Dosing and adverse effects. UpToDate. 2016. Available from: http://www.uptodate.com/contents/directoral-anticoagulants-dosing-and-adverse-effects? source=search_. result\&search=Direct+oral+anticoagulants\&selectedTitle=1\%7E131. Accessed in 2017 (May 15).

3. Kubitza D, Becka M, Mueck W, Zuehlsdorf M. The effect of extreme age, and gender, on the pharmacology and tolerability of rivaroxaban - an oral, direct factor FXa inhibitor. Blood. 2006;108:905. Available from: http:// www.bloodjournal.org/content/108/11/905?sso-checked=true. Accessed in 2017 (May 15)

4. Tripodi A. The laboratory and the new oral anticoagulants. Clin Chem. 2013;59(2):353-62.

5. Baglin T, Hillarp A, Tripodi A, Elalamy I, Buller H, Ageno W. Measuring oral direct inhibitors (ODIs) of thrombin and factor Xa: a recommendation from the subcommittee on control of anticoagulation of the scientific and standardisation Committee of the International Society on thrombosis and haemostasis. J Thromb Haemost. 2013;11:756-760.

6. Tripodi A. Results expression for tests used to measure the anticoagulant effect of new oral anticoagulants. Thromb J. 2013;11(1):9.

7. Gosselin RC, Adcock DM. The laboratory's 2015 perspective on direct oral anticoagulant testing. J Thromb Haemost. 2016;14(5):886-93.

8. Altman R, Gonzalez CD. Simple and rapid assay for effect of the new oral anticoagulant (NOAC) rivaroxaban: preliminary results support further tests with all NOACs. Thromb J. 2014;12(1):7.

9. Douxfils J, Mani $H$, Minet $V$, Devalet $B$, Chatelain B, Dogné JM, et al. Non-VKA oral anticoagulants: accurate measurement of plasma drug concentration. Biomed Res Int. 2015;2015:345138.

10. Gosselin RC, Adcock Funk DM, Taylor JM, Francart SJ, Hawes EM, Friedman $K D$, et al. Comparison of anti-Xa and dilute Russell viper venom time assays in quantifying drug levels in patients on therapeutic doses of rivaroxaban. Arch Pathol Lab Med. 2014;138(12):1680-40.

11. Exner T, Ellwood L, Rubie J, Barancewicz A. Testing for new oral anticoagulants with LA-resistant Russells viper venom reagents. An in vitro study. Thromb Haemost. 2013;109(4):762-5.

12. Douxfils J, Chatelain B, Hjemdahl P, Devalet B, Sennesael AL, Wallemacq P, et al. Does the Russell viper venom time test provide a rapid estimation of the intensity of oral anticoagulation? A cohort study. Thromb Res. 2015;135(5):852-60.
13. Pernod G, Albaladejo P, Godier A, Samama CM, Susen S, Gruel Y, et al Management of major bleeding complications and emergency surgery in patients on long-term treatment with direct oral anticoagulants, thrombin or factor-Xa inhibitors: proposals of the working group on perioperative haemostasis (GIHP) - march 2013. Arch Cardiovas Dis. 2013;106(6-7):382-93.

14. Ebner M, Birschmann I, Peter A, Spencer C, Härtig F, Kuhn J, et al. Point-ofcare testing for emergency assessment of coagulation in patients treated with direct oral anticoagulants. Crit Care. 2017;21(1):32.

15. Li MS, Chapman K, Swanepoel P, Enjeti AK. Sensivity of routine coagulation assays to direct oral anticoagulants: patient samples versus commercial drug-specific calibrators. Pathology. 2016;48(7):712-9.

16. Levy JH, Ageno W, Chan NC, Crowther M, Verhamme P, Weitz Jl. Subcommittee on control of anticoagulation. When and how to use antidotes for the reversal of direct oral anticoagulants: guidance from the SSC of the ISTH. J Thromb Haemost. 2016:14(3):623-7.

17. Steiner $T$, Böhm M, Dichgans M, Diener HC, Ell C, Endres $M$, et al. Recommendations for the emergency management of complications associated with the new direct oral anticoagulants (DOACs), apixaban, dabigatran and rivaroxaban. Clin Res Cardiol. 2013;102(6):399-412.

18. Evans JD. Straighforward statistics for the behavorial sciences. Califórnia: Brooks/Cole Publishing Company; 1996.

19. CLSI. Defining, establishing and verifying reference intervals in the clinical laboratory; approved guideline, third edition. CLSI document C28-A3. Wayne, PA: Clinical and Laboratory Standards Institute; 2008.

20. Mercaldo ND, Lau KF, Zhou XH. Confidence intervals for predictive values with an emphasis to case-control studies. Stat Med. 2007:26(10):2170-83.

21. Baglin T, Keeling D, Kitchen S. British Committee for Standards in haematology. Effects on routine coagulation screens and assessment of anticoagulant intensity in patients taking oral dabigatran or rivaroxaban: guidance from the British Committee for Standards in haematology. $\mathrm{Br} J$ Haematol. 2012;159(4):427-9.

22. Mueck W, Schwers S, Stampfuss J. Rivaroxaban and other novel oral anticoagulants: pharmacokinetics in healthy subjects, specific patient populations and relevance of coagulation monitoring. Thromb J. 2013;11(1):10

23. Francart SJ, Hawes EM, Deal AM, Adcock DM, Gosselin R, Jeanneret C, et al. Performance of coagulation tests in patients on therapeutic doses of rivaroxaban a cross-sectional pharmacodynamic study based on peak and trough plasma levels. Thromb Haemost. 2014;111(6):1133-40.

24. Lippi G, Favaloro EJ. Recent guidelines and recommendations for laboratory assessment of the direct oral anticoagulants (DOACs): is there consensus? Clin Chem Lab Med. 2015:53(2):185-97.

25. Samuelson BT, Cuker A, Siegal DM, Crowther M, Garcia DA. Laboratory assessment of the anticoagulant activity of direct oral anticoagulants: a systematic Review. Chest. 2017;151(1):127-38.

26. Martinuzzo ME, Barrera LH, D'adamo MA, Otaso JC, Gimenez MI, Oyhamburu $J$. Frequent false-positive results of lupus anticoagulant tests in plasmas of patients receiving the new oral anticoagulants and enoxaparin. Int J Lab Hematol. 2014;36(2):144-50.

27. Gosselin R, Grant RP, Adcock DM. Comparison of the effect of the anti-Xa direct oral anticoagulants apixaban, edoxaban, and rivaroxaban on coagulation assays. Int J Lab Hematol. 2016;38(5):505-13.

28. Wong WH, Yip CY, Sum CL, Tan CW, Lee LH, Yap ES, et al. A practical guide to ordering and interpreting coagulation tests for patients on direct oral anticoagulants in Singapure. Ann Acad Med Singap. 2016;45(3):98-105.

29. van Os GM, de Laat B, Kamphuisen PW, Meijers JC, de Groot PG. Detection of lupus anticoagulant in the presence of rivaroxaban using Taipan snake venom time. J Thromb Haemost. 2011;9(8):1657-9.

30. Lalkhen AG, McCluskey A. Clinical tests: sensitivity and specificity. Contin Educ Anaesth Crit Care Pain. 2008;8(6):221-223. Available from: https://

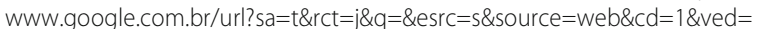
OahUKEwjVyYKvpfLTAhXEg5AKHQTiCRYQFggpMAA\&url=http\%3A\%2F\%2 Fserene.me.uk\%2Fhelpers\%2Fsensitivity-specificity.pdf\&usg=AFQjCNFKq268cIQ1 -Hi7tOSRB7ergjDvuw. Accessed in 2017 (May 15). 